\title{
TRANSCATHETER VERSUS SURGICAL AORTIC VALVE REPLACEMENT: FIRST EXPERIENCE WITH A NEW TAVI SYSTEM
}

DOI: $10.36740 /$ WLek202104108

\author{
Glib I. Yemets, Oleksandra V. Telehuzova, Andrii V. Maksymenko, Georgiy B. Mankovsky, Yevhen Y. Marushko, \\ Arkadii A. Dovgalyuk, Illya M. Yemet \\ UKRAINIAN CHILDREN'S CARDIAC CENTER, KYIV, UKRAINE
}

\begin{abstract}
The aim: to reveal early results after transapical TAVI with a new self-manufactured XPand system, comparing them with SAVR and common transfemoral TAVI outcomes. Materials and methods: Eighty-four patients (mean age 79,5 $\pm 10,2$ years) with severe aortic stenosis were operated on from January 2016 to February 2019 . Nine patients had undergone the TAVI (two with transfemoral access route and seven with transapical, using the XPand system). SAVR was performed in seventy five patients. For the latter, we estimate the in-hospital mortality, complication rates, intensive care unit and total hospital length of stay.

Results: There was no intraoperative mortality. In the TAVI group, the frequency of intraoperative and postoperative complications was significantly lower ( $p<0.01)$. The SAVR group showed higher median intensive care unit length of stay (104h, IQR $72-112$ versus $29 \mathrm{~h}$, IQR $20-35, \mathrm{p}<0.01)$, hemodynamic support duration ( $100,98 \pm 78$ minutes versus $11.13 \pm 7.89$ minutes, $p<0.01)$ and paravalvular leakage causality $(9,33 \%$ versus $0 \%)$. No significant difference in results depending on the TAVI access routes was obtained. Conclusions: We conclude that TAVI provides an alternative to the conventional approach in patients with severe aortic stenosis aged over 75 years. No significant difference in mortality rate between TAVI and SAVR groups was found. A novel transapical TAVI device is associated with good short-term results and lower complication rate.
\end{abstract}

KEY WORDS: transcatheter aortic valve replacement, TAVI, SAVR, aortic stenosis, elderly patients, XPand

Wiad Lek. 2021;74(4):856-863

\section{INTRODUCTION}

Cardiovascular disease (CVD) is of interest because supposed to be the most common source of adult morbidity and mortality for many decades [1]. Among the pathology variety, leading position is traditionally occupied by the coronary heart disease, acquired valvular heart disease and other conditions provokingthe life expectancy reduction, especially in the elderly [2 ]. With a dramatic geriatric population life expectancy increase in Western Europe and North America, acquired defects due to degenerative valve changes, came to the fore in the valvular pathology structure beside adults. These rapid changes are having a serious effect on treatment approaches and strategies, pushing the process of knowledge producing.

Nevertheless, degenerative aortic stenosis (AoS) is the most common valve disease in adults, whose prevalence increases with age, affecting approximately $4 \%$ of individuals older than 75 years [3]. A primary concern of AoS is unfavourable prognosis with symptoms manifestation and conservative treatment. Therefore, the average life expectancy after the anginal attacks, syncope or heart failure appeared in these patient groups is only 5, 3 and 2 years, respectively [4].

Surgical aortic valve replacement (SAVR) is a traditional method which has considerable impact on life quality and duration. However, approximately $30 \%$ of patients with AoS and surgical indication are not submitted to valve replacement due to advanced age, presence of comorbidities, high surgical risk etc. Osama Alsara et. al [5] notes that advanced age has been a leading barrier to surgical intervention in elderly patients based on the increasing with age peri-operative mortality rate from $1.3 \%$ in patients $\leq 70$ years old, to about $5 \%$ at age $80-85$ years. Aforementioned statements turning out the benefit / risk surgical replacement ratio for being controversial.

More than a decade ago, the transcatheter aortic valve implantation (TAVI) was offered as an alternative option. Describing this procedure, we have to admit that traditionally prosthetic valve insertion is performed through the vascular access and withdraw the need for open heart surgery (artificial circulation and cardiac arrest are not required). Thus, the periprocedural death, risk of bleeding and other complications could be reduced. Although TAVI is widely used for symptomatic severe aortic stenosis in patients over 75 in Europe and North America. Alas, in Ukraine, this procedure had been performed only in a few isolated cases [6].

As discussed earlier, to manage the severe aortic stenosis two major approaches are usually applicable: TAVI and SAVR. These methods have achieved significant improvements in survival rates as well as other outcomes in patients with severe aortic stenosis [7]. However, approximately $5.2 \%$ among elderly patients with symptomatic severe aortic stenosis undergoing surgical aortic valve 
Table I. Baseline patients characteristics in both groups

\begin{tabular}{|c|c|c|c|}
\hline & TAVI (mean \pm SD) & SAVR (mean $\pm S D$ ) & p-value \\
\hline Number & 9 & 75 & \\
\hline Age,years & $81 \pm 8,2$ & $78.53 \pm 1.7$ & $<0.01$ \\
\hline Male & $1(11.1 \%)$ & $40(53.3 \%)$ & $<0.001$ \\
\hline Coronary Heart Disease & $5(55.5 \%)$ & $63(84 \%)$ & $<0,05$ \\
\hline Multivascular lesions & 0 & $45(60 \%)$ & $<0.001$ \\
\hline NYHA III, IV & $8(88.9 \%)$ & $54(72 \%)$ & $<0,01$ \\
\hline AF before surgery & 0 & $15(20 \%)$ & $<0,001$ \\
\hline COPD & 0 & $4(5.3 \%)$ & $<0.05$ \\
\hline Diabetes & $2(22.2 \%)$ & $30(40 \%)$ & $<0.05$ \\
\hline EuroScore II & $9.79 \pm 5.1$ & $9.41 \pm 4.06$ & $=0.1$ \\
\hline STS Score & $7.85 \pm 2.35$ & $7.62 \pm 3.24$ & $=0.1$ \\
\hline
\end{tabular}

replacement are in high-risk and in $80 \%$ cases they meet the criteria for TAVI. The distribution of these indicators to the general population in Western Europe and North American countries shows that approximately 290 thousand adults in mentioned regions are potential candidates for TAVI [6,7]. Extrapolating given by Osnabrugge et al [8] data to the Ukrainian real life population over 75 (rough quantity in 2013 was about 3 million), the number of symptomatic aortic stenosis patients considered to be candidates for TAVI could vary to 14 thousands. These theses highlight the importance of transcatheter aortic valve replacement in Ukraine.

Annually, we perform more than 25 procedures on patients with aortic stenosis over 75 in our Clinic. There is a growing body of literature that recognises the importance of managing these patients primarily associated with comorbidity and severe general condition, therefore with a very high risk of surgery due to the artificial circulation [9]. The specific objective of this study was to present the primary data pool on the aortic stenosis treatment by both TAVI and SAVR, and to analyze the immediate and long-term outcomes of described methods. Obtained data should be generalized, according to the hight external validity.

\section{THE AIM}

The aim was to reveal early results after transapical TAVI with a new self-manufactured XPand system, comparing them with SAVR and common transfemoral TAVI outcomes.

\section{MATERIALS AND METHODS}

Study was performed according to the Good Clinical Practice (GCP) standards and World Medical Association ( WMA ) Helsinki Declaration. Prior to participation and data collection, all subjects received an explanation of the project and signed the inform consent.

An observational and longitudinal study was carried out at Ukrainian Children's Cardiac Center. Clinic for Adults
(UCCC) located in Kyiv. We include all patients over 75 with severe, symptomatic AoS, submitted to TAVI or SAVR, from January 2016 to February 2019. The participant's group was representative with respect to gender and age. In order to achieve valid results, we analysed anamnesis of patients, treatment protocols and early postprocedural follow - ups of 84 aortic valve replacement operations. Preprocedural data included the clinical examination and complementary tests (resting electrocardiography, chest $\mathrm{X}$-ray, transthoracic Doppler echocardiography with protocol for aortic complex measurements; computed tomography (CT) angiography of the heart and total aorta, and cardiac catheterization with coronary angiography). Transesophageal echocardiography was used to guide the procedure and detect possible complications due to prosthesis implantation. In some cases, additional tests were performed to rule out or confirm comorbidity (MRI of the abdominal cavity, etc.). Patients, undergoing TAVI were additionally examined by MRI with contrast to assess femoral and superficial arteries. Among the analyzed cases, 75 procedures $(89.28 \%)$ were SAVR and $9(10,7 \%)-$ TAVI.

\section{ELIGIBILITY CRITERIA}

Inclusion criteria for transcatheter aortic valve implantation were: (1)severe aortic stenosis, (2) valve's calcification or fibrosis and (3) age $>75$ years.

Exclusion criteria were: (1) multivessel hemodynamically significant coronary artery disease required surgical correction, (2) the distance from the aortic valve fibrous ring to the coronary arteries less than $10 \mathrm{~mm}$, (3) severe mitral and tricuspid insufficiency.

\section{GROUP CHARACTERISTICS}

A number of patients with isolated aortic stenosis in TAVI group was 4 (44.4\%). Just over half the sample $(55,6 \%)$ was diagnosed with coronary heart disease, which is considered to be an aggravating factor and adversely affects the life expectancy prognosis. In SAVR group, 63 patients $(84 \%)$ 
were diagnosed with coronary heart disease and 24 patients (32\%) with type II diabetes mellitus. The chronic obstructive pulmonary disease was diagnosed in 4 patients (5\%). The primary baseline data are given in Table I.

The risk of surgery using EuroSCORE II and STS was calculated for each patient and showed higher values in TAVI group. The statistical significance was not estimated. However, each risk stratification model suffers from limitations and still not perfect [10]. Baseline echocardiographic parameters are shown in Figure 1.

\section{STATISTICAL ANALYSIS}

Continuous variables were described as mean and standard deviation, and categorical variables as absolute number and percentage. Statistical significance was analysed using analysis of variance and t-tests as appropriate. Student's t-test (or the Mann-Whitney test) was used to compare continuous variables for independent samples, and the chi-squared test or Fisher's exact test was used for categorical variables, as appropriate. To assessif the means of two groups are significantly different the ANOVA was used. All analyses were performed using Stata IC and Excel software version. Statistical significance level was set at 5\%.

\section{PATIENT PREPARATION AND TYPE OF PROSTHESIS USED}

All TAVI patients were pretreated with $100 \mathrm{mg}$ acetylsalicylic acid and $300 \mathrm{mg}$ clopidogrel on the day prior to implantation. In patients with renal dysfunction, intravenous hydration with $0.9 \%$ saline solution at a dose of 0.3 to $0.5 \mathrm{~mL} / \mathrm{kg} /$ hour was started 12 hours before the procedure.

The self-expanding XPand prosthesis was used for transapical access. Patients were admitted to the intensive care unit after the intervention, and on the first day after the procedure, underwent laboratory tests, electrocardiogram, and echocardiogram.

\section{SURGICAL PROCEDURES}

\section{TAVI}

The procedures were performed in a hybrid operating room (HOR) under general anaesthesia. Two access routes for TAVI implantation were performed: transapical andtransfemoral (7 procedures and 2 procedures, respectively) with mean duration $173.3 \pm 35.5 \mathrm{~min}$.

Essential Key Steps:

- To evaluate and confirm the transcatheter heart valve size by CT and ECHO

- To study the deployment side (comparing right or left femoral artery)

- Vascular access

- Place the transvenous right ventricle pacing catheter

- Sheath insertion

- To place the diagnostic pigtail catheter in the non-coronary cusp
- Establish coplanar view

- Cross the native valve

- To assess and stabilise hemodynamics

- To place the transcatheter heart valve

- Assess placement at 2/3 deployment (valve can be recaptured and repositioned at this point)

- Transcatheter heart valve full deployment

- Measuring the post-deployment

- Device and sheath removal

- Vascular closure

Transfemoral TAVIs were performed under the general anaesthesia with usage both fluoroscopic and ultrasound guidance to ensure the optimal percutaneous stick. Transfemoral arterial and venous accesses were obtained with $18 \mathrm{~F}$ introducer, subsequently introducing the $J$-shaped wire and a pigtail catheter positioned in the ascending aorta. Safari wire was exposed to the left ventricle. The valve delivery system was loaded onto the guidewire with the flush ports pointing up. An angiogram was obtained to determine the appropriate TAVI deployment angles. Once the valve delivery system was brought into appropriate implanting position (depth of 3-5 mm), a coplanar image projection was achieved to see the radiopaque marker as a straight line. Additionally, the latter is preferred if both cannot be achieved together. In all cases of transfemoral implantation, we got good procedural outcome without complications.

Transapical TAVIs were done by the left-side thoracotomy through a 4 -inch incision between the ribs. The left ventricular (LV) apex was exposed using a limited left anterolateral thoracotomy via ultrasound control. To deliver LV stimulation, the cathode of an external pacemaker was attached to the distal external end of the guidewire using a crocodile clip. A double purse-string suture with Teflon was placed into the left avascular zone. We punctured a LV apex with6F introducer, then inserted a soft conductor through the aortic valve to abdominal aorta bifurcation. The left ventricular apex was accessed, and a stiff wire was placed across the aortic valve to insert the $22 \mathrm{~F}$ delivery sheath. A system for aortic valve ballooning was exposed via wire. Valve unfolding was carried out by transcatheter introduction of a balloon or self-expandable valve under high rate ventricular pacing (160-170 bpm) aimed to reduce heart movement. Heart Team assessed the intraoperative graft position, coronary arteries permeability and aortic valve function via angiography and ultrasound. Finally, the guidewire was removed, the sutures locked and cut.

In all transapical aortic valve implantation cases we used the self-expandable novel XPand prosthesis, created in collaboration between German, French and Ukrainian scientists. Device consists of two parts: (1) tubular slotted stainless-steel stent frame with unidirectional trileaflet bovine pericardial tissue and a (2)fabric skirt.Trileaflet bovine pericardial tissue sutured into a wire frame of nitinol, a nickel (55\%)-titanium (45\%) alloy that has temperature-associated shape memory features.

Currently, XPand device could be used only for the transapical access. It has been second standard approach 


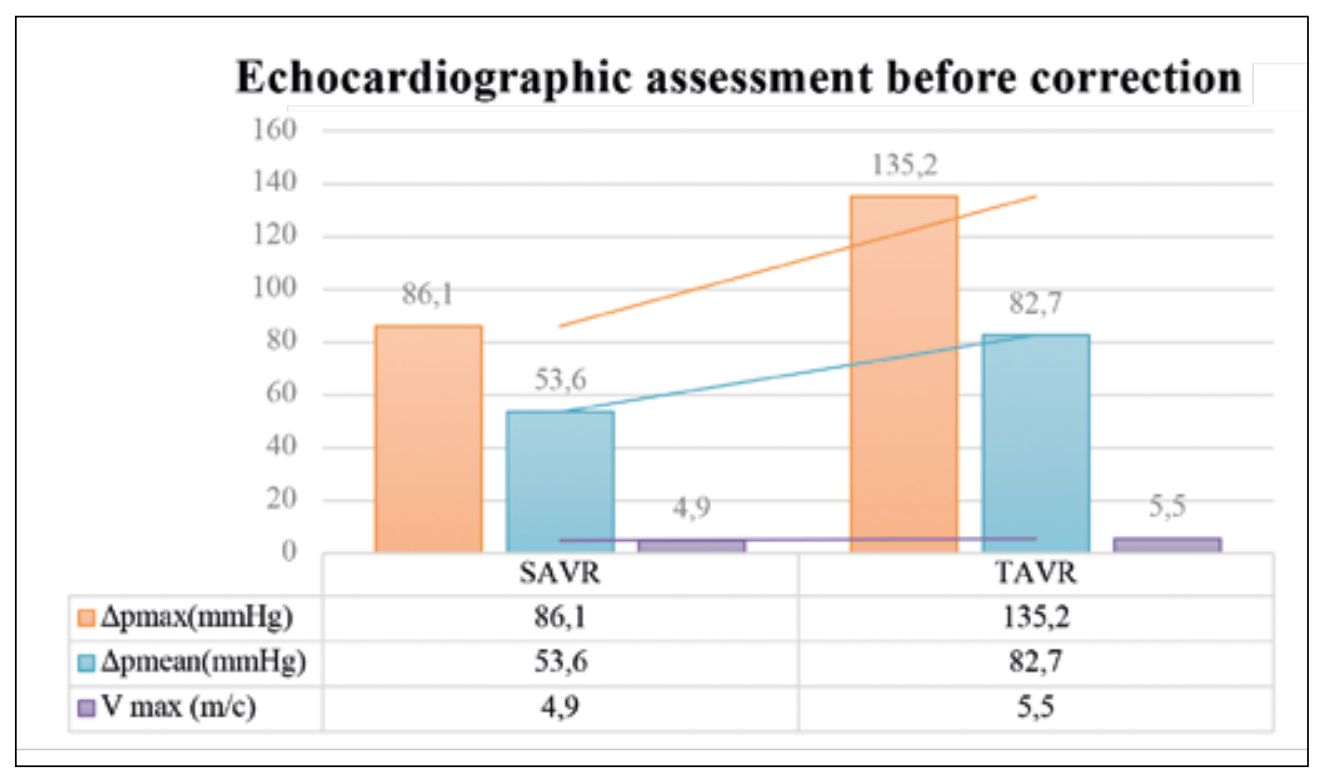

Fig. 1. Basic echocardiographic assessment in both groups before correction

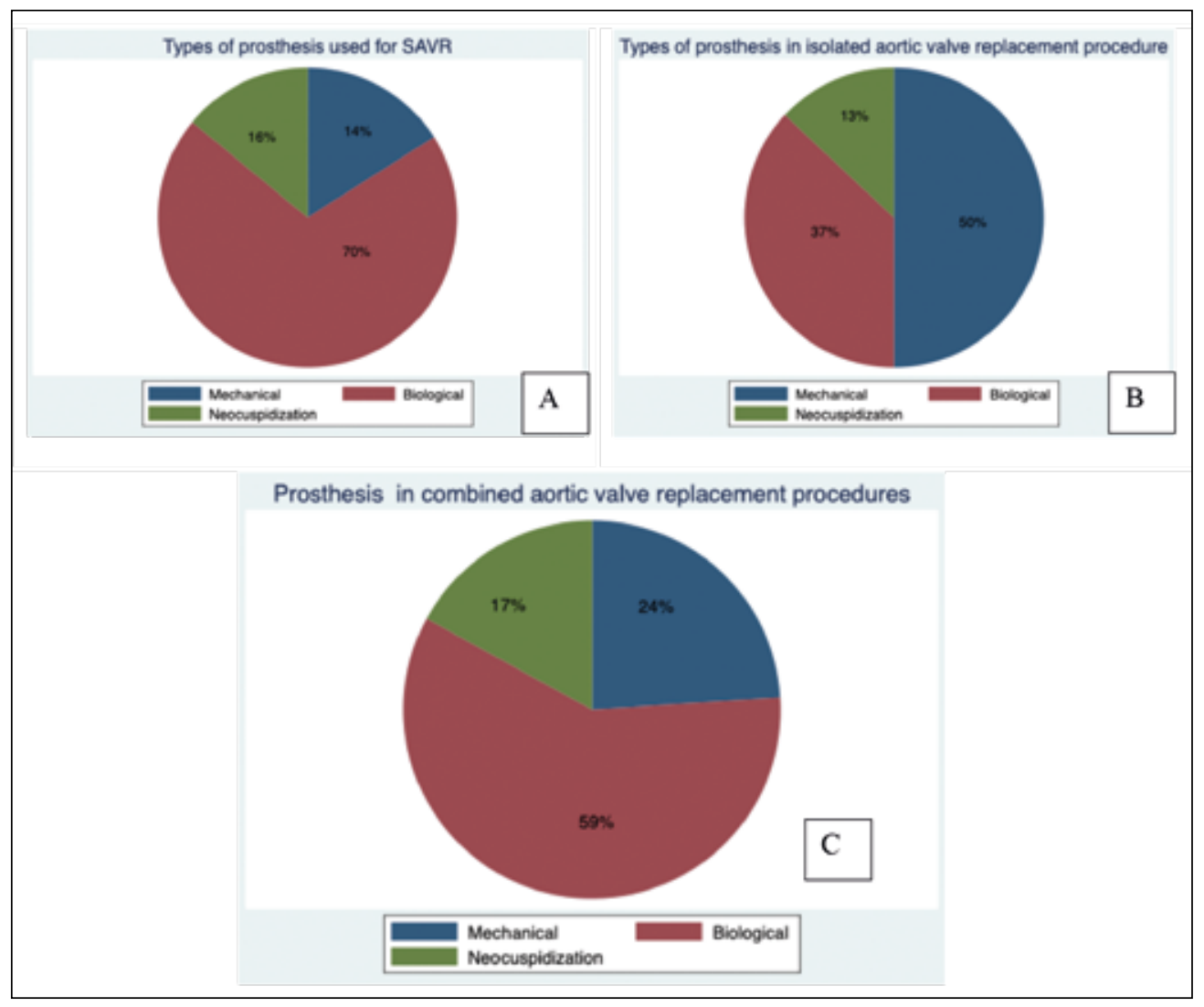

Fig. 2. Types of prosthesis used in SAVR group: . A - General data, B - For isolated aortic valve replacement, C - For combined procedures

after transfemoral, with stable rates although the advancement of alternative options [12]. Transapical access, aside from the ability to deliver a large device in a patient with inadequate iliofemoral vessels, may offer additional advantages in complex patients. Undoubtable advantage of this approach is the shortest and direct access to the aortic valve, which makes the procedure of prosthesis delivery and positioning more controllable.
SAVR

The procedures were performed with cardiopulmonary bypass (CPB) and moderate hypothermia (28-32o C) using anterograde crystalloid cardioplegia for myocardial protection. Patients underwent surgery through a median sternotomy, the heart was arrested after cardiopulmonary bypass. The participants were divided into two groups based on the type of procedure: isolated aortic valve replacement $(n=15)$ 


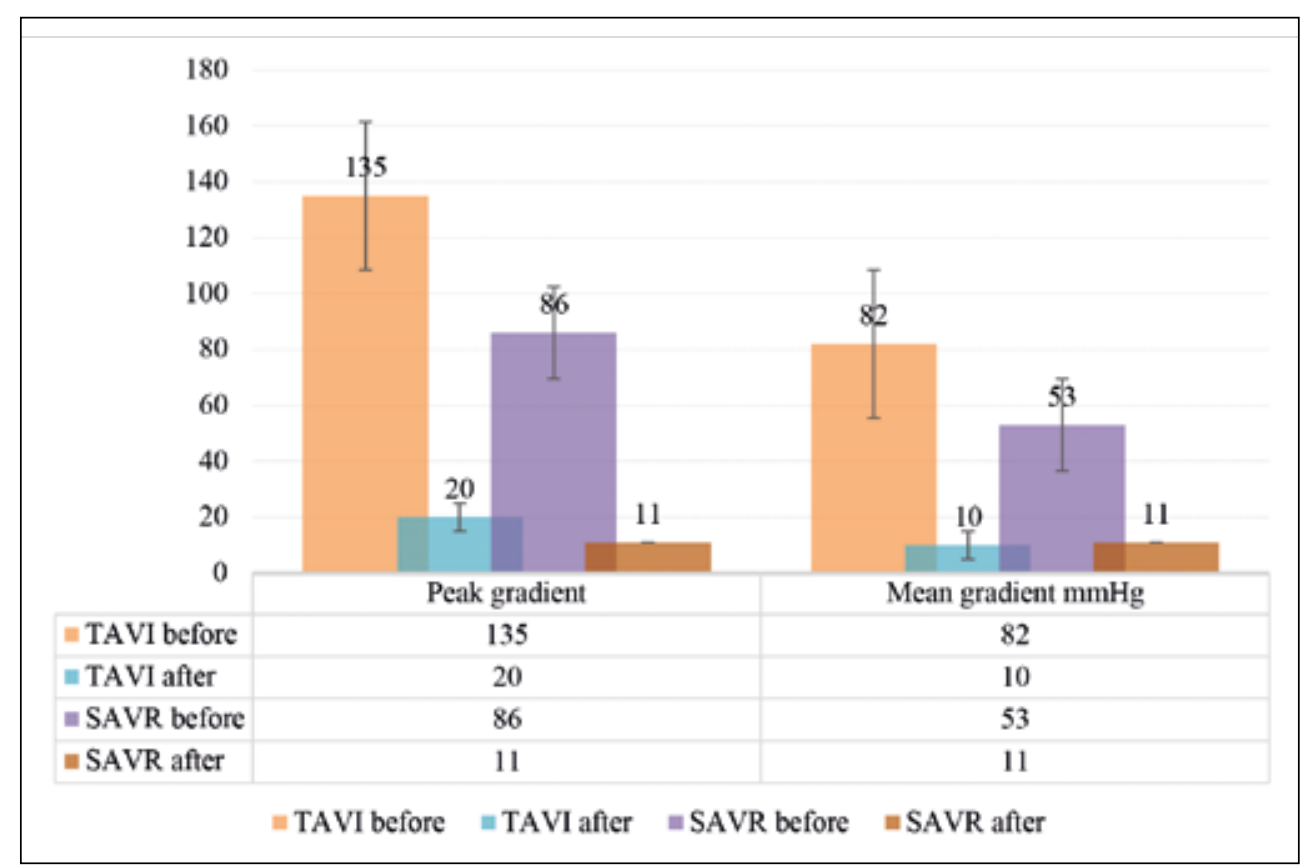

Fig. 3. Echocardiographic assessment before and after procedures

and coronary artery bypass grafting (CABG) with aortic valve replacement $(n=41)$. The mean number of bypasses in combined procedures was $2.87 \pm 0.66$ per patient. We performed aortic root enlargement procedure described by Nicks et. al for five patients (6,67\%). In52 (69,3\%) cases AV valve was replaced with biological prosthesis, in 12 cases $(17,4 \%)$ - with mechanical prosthesis and in ten cases $(13,3 \%)$ aortic valve neocuspidization with autologous pericardium was performed. Figure 2 presents the results obtained from the preliminary analysis of SAVR group.

\section{RESULTS}

\section{PRIMARY OUTCOMES}

It should be noted that all patients had a good postprocedural outcomes, confirmed by the obtained data. No critical complications requiring additional treatment (eg, ECMO and dialysis) have been identified.Strong evidence was found when comparing transfemoral versus transapical TAVI. Both accesses showed good results with no difference in 30-day mortality rates and ultrasound characteristics between groups. This is a remarkable result to conclud that transapical could be an alternative option for transfemoral access.

If we now turn to the average length of stay (ALOS) (from admission to discharge, transfer ) ranged as 21.35 \pm 8.1 days. The most striking result to emerge from the data is that average ICU length of stay (LOS), time of the artificial ventilation (ventilation) and duration of inotropic support were significantly lower in TAVI group. The differences between groups outcomes and complications are highlighted in Table II and III, respectively.

The next section of the survey was concerned with procedure time, which was significantly lower in TAVI group-
$173.3 \pm 22.4$ min versus $226.7 \pm 31.5$ minin SAVR groupand duration of anaesthesia: $354.3 \pm 26.5 \mathrm{~min}$ in TAVI group and $428 \pm 30.4 \mathrm{~min}$ in SAVR group. The mean intraoperative bleeding volumewas significantly lower in TAVI group, $208.8 \pm 13.8 \mathrm{ml}$ comparing with $978.5 \pm 45.6 \mathrm{ml}(\mathrm{p}<0.01)$ in SAVR group.

Echocardiographic assessment of the procedure effect reliedon peak velocity, mean pressure gradient (MPG), aortic valve area (AVA) and left ventricle ejection fraction (EF). In the current study, comparing preprocedural echocardiographic assessment with postprocedural showed that the mean value of positive changes did not show any significant difference between TAVI and SAVR groups. The results obtained from the preliminary analysis are set out in Figure 2.

The single most striking observation to emerge from the data comparison was low level of paravalvular insufficiency in both groups. A minority of subjects in TAVI group $(20,4 \%)$ indicated the minimal paravalvular insufficiency. However, $9 \%$ of patients in the surgical group were diagnosed with moderate paravalvular insufficiency, while patients in the TAVI group had no data for moderate paravalvular insufficiency. Figure 3.

Interestingly, there were also differences in the ratios of postcardiotomy syndrome frequency between two groups $(p<0.01)$. Finally, important clinically relevant finding was that the postcardiotomy syndrome was mild and did not require additional manipulations (eg, pericardial drainage) in the TAVI group.

The syndrome was characterized by pleural effusions (up to $20 \mathrm{~mm}$ ), increased levels of C-reactive protein and subfebrile, which were cured conservative. In contrast, in SAVR subjects, the duration of postcardiotomy syndrome and its severity prevailed over those in TAVI patients. The syndrome was also characterized by pericardial or pleuritic 
Table II. Primary postprocedural outcomes in the TAVI and SAVR groups

\begin{tabular}{|c|c|c|c|}
\hline & TAVI & SAVR & p value \\
\hline Surgery duration, $\min$ & $173.3 \pm 35,5$ & $354.2 \pm 113.6$ & $<0,001$ \\
\hline Average length of stay, days & $24.1 \pm 2.4$ & $18.6 \pm 3.2$ & $<0.05$ \\
\hline Average stay duration in ICU, days & $1.29 \pm 0.5$ & $4.75 \pm 2.89$ & $<0.01$ \\
\hline Ventilation duration, hours & $12.01 \pm 7.2$ & $19.35 \pm 9.68$ & $<0.05$ \\
\hline Hemodynamic support duration, hours & $11.13 \pm 7.89$ & $100,98 \pm 78,99$ & $<0.001$ \\
\hline
\end{tabular}

Table III. Mortality rates and complications in the TAVI and SAVR groups

\begin{tabular}{cccc}
\hline & TAVI & SAVR & P value \\
\hline 30-day mortality rate & 0 & $3(4 \%)$ & $<0,1$ \\
\hline Intraoperative blood loss, $\mathrm{ml}$ & $208.8 \pm 81.6$ & $978.5 \pm 230.3$ & $<0.01$ \\
\hline Blood loss by drainages, $\mathrm{ml}$ & $130.5 \pm 34.7$ & $331.72 \pm 120.8$ & $<0.01$ \\
\hline Resternotomy & 0 & $4(5.3 \%)$ & $<0.05$ \\
\hline Pneumonia & 0 & $9(12 \%)$ & $<0,001$ \\
\hline Atrial fibrillation & $3(33.3 \%)$ & $30(40 \%)$ & $<0.01$ \\
\hline Paravalvular leakage & 0 & $7(9,33 \%)$ & $<0.01$ \\
\hline Postpericardiotomy syndrome & $2(22.2 \%)$ & $23(31.3 \%)$ & $<0.01$ \\
\hline EX implantation & $1(11.1 \%)$ & $2(2.7 \%)$ & $<0.05$ \\
\hline Stroke & $1(11.1 \%)$ & 0 & $<$ \\
\hline Sepsis & 0 & $5(6.7 \%)$ & $<0.1$ \\
\hline
\end{tabular}

pain, friction rubs, pleural effusions, pneumonitis, and abnormal ECG and radiography findings, required surgical manipulations ( $8 \%$ of patients underwent pericardial drainage). Surprisingly, TAVI was less common found to cause the atrial fibrillation - $33.3 \%$ compared to $46.3 \%$ in the SAVR group ( $\mathrm{p}<0.01)$.

These findings will doubtless be much scrutinised, but there are some immediately dependable conclusions for 30-day mortality rates in patients of high surgical risk over 75 , which didn't exceed $4 \%$ in our study. Recently, we can report the low mortality rate and it is apparent from the data, that 30-day mortality rate in TAVI group was 0 cases. The follow-up results showed that the annual mortality rate in patients after surgical aortic valve prosthesis was $2.6 \%$ (the cause of death was fatal comorbidities not related to thecardiovascular system).

The results in this chapter indicate that 12 months follow-up outcomes and survival analysis showed $2,6 \%$ mortality rate in SAVR group (however, due noncardiovascular diseases), simultaneously in TAVI group absence of death events was reported. The next chapter, therefore, moves on to discuss the pros and cons of TAVI for high risk patients over 75 .

\section{DISCUSSION}

The empirical findings in this study provide a new understanding of TAVI and SAVR advantages. However, TAVI is an evolving modality currently available for patients with severe AS who are unsuitable for surgery because of technical/anatomical issues or too high-estimated surgical risk. This new understanding should help to improve predictions of the impact of transcatheter aortic valve implantation and to form generally new approach in AS treatment. The present study adds to the growing body of research that indicates TAVI competitive advantages in high surgical risk patients aged over 75 , contemporaneously the "golden standard" - surgical valve replacement.

Prior studies that have noted the potential benefits of TAVI $[14,15]$, taken together, results of our study suggest that this method provides less blood loss and complications, simultaneously reducing average stay duration in ICU.

Our study will prove useful in expanding the understanding of alternative to SAVR approach, which is the intervention of choice for older patients worldwide. The scope of this study was limited in terms of TAVI novelty for Ukraine, nevertheless, we were able to establish that both methods were safe and effective, as far as no significant difference in overall mortality rates between TAVI and SAVR was found. These results corroborate the findings of a great deal of the previous meta-analyzes $[5,8,9]$.

According to the SURTAVI and PARTNER IIA trials data, we can infer that safety and efficacy of TAVI approach indicating the widespread use of this technique among high-risk patients $[6,7]$. 
Our study found that open surgical aortic valve replacement was associated with a higher surgery duration, increased bleeding risk and incidence of postoperative complications (pneumonia, postpericardiotomy syndrome and acute renal failure).

Several questions still remain to be answered, although the findings of this study have a number of practical implications.We found numerous advantages for TAVI in elderly patients:

1. Reduction of Intensive Care Unit length of stay.

2. Minimize blood loss.

3. Reduction of surgery duration.

4. Artificial blood circulation is not required.

5. Possibilities to perform TAVI in patients of high surgical risk with severe comorbidities and patients required re-intervention.

This research has thrown up many questions in need of further investigation.Numerous clinical challenges including paravalvular insufficiency, vascular complications, coronary artery occlusion, valve ring rupture, ventricular perforation, stroke etc. still remain unsolved. A reasonable approach to tackle this issue could be to manage this group of patients by the Heart Team [16].

Notwithstanding the relatively limited sample, this work offers valuable insights into the clinical features of AS patients over 75, presenting a novel XPand device. According to the high cost of TAVI, and therefore limited number of procedures associated with the rapidly increasing number of patients, Ukrainian scientists have developed a modern aortic valve biological prosthesis and delivery system. Undoubtedly, this invention is able to solve many issues with consistently high quality and reliability.

\section{CONCLUSIONS}

1. No significant difference in mortality rate between TAVI and SAVR groups was found.

2. The frequency of complications, including pneumonia $(\mathrm{p}=0.001)$ and postcardiotomy syndrome $(\mathrm{p}=0.01)$ was lower in TAVI group.

3. There was a statistically significant Reduction of Intensive Care Unit length of stay in TAVI group $(<0.01)$.

4. No statistically significant difference between echocardiographic assessment was detected.

5. AF in TAVI patients has been significantly lower $(\mathrm{p}<0.01)$.

\section{REFERENCES}

1. Mallis P., Michalopoulos E., Dimitriou C. et al. Histological and biomechanical characterization of decellularized porcine pericardium. Bio-Medical Materials and Engineering. 2017; 28:477-488.

2. Hughes G.H., Cutter G., Donahue R. et al. Recruitment in the Coronary Artery Disease Risk Development in Young Adults (Cardia) Study. Control Clin Trials. 1987;8:68S-73S.

3. Vahanian A., Alfieri 0., Andreotti F. et al. Joint Task Force on the Management of Valvular Heart Disease of the European Society of Cardiology (ESC); European Association for Cardio-Thoracic Surgery (EACTS). Guidelines on the management of valvular heart (version 2012). Eur Heart J. 2012;33(19):2451-96.
4. Townsend C.M. Jr., Beauchamp R.D., Evers Mattox K.L. Sabiston textbook of surgery:the biological basis of modern surgical practice. Philadelphia. 2008;18.

5. Isarah A., Alsara 0., Bachauwa G. Hypertension Management in the Elderly: What is the Optimal Target Blood Pressure? Heart Views. 2019;20(1) 11-16. doi:10.4103/heartviews.heartviews_28_18.

6. Siontis G.C.M., Overtchouk P. , Cahill T.J. et al. Transcatheter aortic valve implantation vs. surgical aortic valve replacement for treatment of symptomatic severe aortic stenosis : an updated meta-analysis. Eur Heart J.2019 0ct 7;40(38):3143-3153. doi: 10.1093/eurheartj/ehz275.

7. Eghbalzadeh K., Rahmanian P.B., Giese D., Kuhn E.W. SAVRversus TAVl:What about the Hemodynamic Performance? An InVivo and In Vitro Analysis. 2019.

8. Osnabrugge R.L., Kappetein A.P., Reynolds M.R., Cohen D.J. Costeffectiveness of transcatheter valvular interventions: economic challenges. 2013.

9. Baumgartner H., FalkV., Bax J.J. et al. 2017 ESC/ EACTS Guidelines for the management of valvular heart disease. Eur Heart J. 2017;38:2739-91.

10. Khan A.A., Murtaza G., Khalid M. F., Khattak F. Risk Stratification for Transcatheter Aortic Valve Replacement. Cardiology research. 2019;10(6):323-330. https://doi.org/10.14740/cr966.

11. Reardon M.J., VanMieghem N.M., Popma J.J. et al. Surgicalortranscatheteraortic-valvereplacementinintermediateriskpatients. N EnglJ Med. 2017;376:1321-31. https://doi.org/10.1056/ NEJMoa 1700456.

12. Ferrari E., Eeckhout E., Keller $S$. et al. Transfemoral versus transapical approach for transcatheter aortic valve implantation: hospital outcome and risk factor analysis.J Jardiothorac Surg. 2017;12(1):78. doi:10.1186/ s13019-017-0638-9.

13. Nishimura R.A., Otto C.M., Bonow R.0. et al. 2017 AHA / ACC focused update of the 2014 AHA / ACC guideline for the management of patients with valvular heart disease: a report of the American College of Cardiology / American Heart Association Task Force on Clinical Practice Guidelines. J Am Coll Cardiol. 2017, 70: 252-89.

14. Gargiulo G., Sannino A., Capodanno D. et al. Transcatheter aortic valve implantation versus surgical aortic valve replacement: a systematic review and meta-analysis. Ann Intern Med. 2016; 165:334-44. https:// doi.org/10.7326/M16-0060.

15. Cao C., Ang S.C., Indraratna P. et al. Systematic review and metaanalysis of transcatheter aortic valve implantation versus surgical aortic valve replacement for severe aortic stenosis. Annals of Cardiothoracic Surgery. 2013; 2: 10-23.

16. Panchal H.B., LadiaV., Amin P. etal. A meta-analysis of mortality and major adverse cardiovascular and cerebrovascular events in patients undergoing transfemoral versus transapical transcatheter aortic valve implantation using Edwards valve for severe aortic stenosis. Am J Cardiol. 2014; 114: 1882-90. https://doi.org/10.1016/j.amjcard.2014.09.029.

\section{ORCID and contributionship:}

Glib I.Yemets: 0000-0002-6139-6235 A,B,D,F

Oleksandra V. Telehuzova: 0000-0003-4801-093X $X^{B, C, D}$

Georgiy B. Mankovsky: 0000-0003-0335-7868 A,E

Andrii V. Maksymenko: 0000-0002-6615-9036 E,F

Yevhen Y. Marushko: 0000-0002-0696-9926 ${ }^{E}$

Arkadii A Dovgalyuk: 0000-0002-6606-394X ${ }^{A, E}$

Illya M. Yemets: 0000-0002-1893-0164 A,E,F

\section{Conflict of interest:}

The Authors declare no conflict of interest. 


\section{CORRESPONDING AUTHOR}

Oleksandra V. Telehuzova

Ukrainian Children's Cardiac Center.Clinic for Adults

24 Illienka st., 04050 Kyiv, Ukraine

tel: +380687044822

e-mail: teleguzova5@gmail.com

Received: 06.11 .2020

Accepted: 04.03 .2021

A - Work concept and design, B - Data collection and analysis, C - Responsibility for statistical analysis,

$\mathbf{D}$-Writing the article, $\mathbf{E}$-Critical review, $\mathbf{F}$ - Final approval of the article 